\title{
Inversión social responsable: una forma de potenciar los agronegocios - caso Colombia
}

\section{Socially responsible investment: a way to impulse agribusinesses - the case of Colombia}

\author{
Orlando E. Contreras-Pacheco ${ }^{1}$; Eduardo Carrillo Zambrano ${ }^{2}$
}

1'Ing. Industrial, MBA., Ph.D.(c). Universidad Industrial de Santander, Bucaramanga, Santander, Colombia; e-mail: ocontrer@uis.edu.co; Dhttps://orcid. org/0000-0002-3584-7063

${ }^{2}$ Ing. de Petróleos, M.Sc., Ph.D. Universidad Autónoma de Bucaramanga, Bucaramanga, Santander, Colombia; e-mail: ecarrill@unab.edu.co; Dhttps://orcid. org/0000-0002-0868-940X

Cómo citar: Contreras-Pacheco, O.E.; Carrillo Zambrano, E. 2019. Inversión social responsable: una forma de potenciar los agronegocios - caso Colombia. Rev. U.D.C.A Act. \& Div. Cient. 22(2):e1287. http://doi.org/10.31910/rudca.v22.n2.2019.1287

Artículo de acceso abierto publicado por Revista U.D.C.A Actualidad \& Divulgación Científica, bajo una licencia Creative Commons CC BY-NC 4.0

Recibido: 21 de junio de 2018

Aceptado: 9 de julio de 2019

Editado por: Ingeborg Zenner de Polanía

\section{RESUMEN}

El presente trabajo busca establecer la forma en que la inversión social responsable tiene el potencial de dinamizar el desarrollo empresarial de un país y, al mismo tiempo, asumir los objetivos de sostenibilidad, como propósitos propios de las actividades productivas en los agronegocios. Para ello, se propone una aproximación a un modelo teórico ideal, que expone la dinámica de la inversión social responsable, como impulsor del desarrollo empresarial. En lo metodológico, mediante el análisis de la literatura existente, se estudia el fenómeno desde la perspectiva teórica y, posteriormente, desde la perspectiva empírica, se hace uso de un análisis de framework sobre siete casos de estudio colombianos. De esta forma, se abordan conceptos relacionados con el desarrollo empresarial y la influencia de modelos de agronegocio de doble y triple impacto, así como en factores condicionantes para el otorgamiento de la figura de financiación respectiva. A manera de resultados, junto al modelo teórico expuesto, se suministra un planteamiento basado en cinco proposiciones de asociación, lo cual, prepara el terreno para su futura comprobación deductiva, tanto cualitativa como cuantitativamente.
Palabras clave: emprendimiento responsable; inversión social responsable; red colaborativa de emprendimiento; sostenibilidad.

\section{ABSTRACT}

This work aims to determine the way in which social responsible investments has the potential of providing dynamism to the entrepreneurial development in agribusiness initiatives. At the same time, it seeks to integrate this concept with sustainable development objectives, and with conventional financial purposes within this particular industry. To do it, this work proposes an ideal theoretical model that exposes the dynamics of the social responsible investments and its positive influence on the entrepreneurial development. Methodologically, by using relevant literature, a conceptual analysis is performed from a theoretical perspective, followed by a framework analysis through the lens of seven Colombian case studies in order to perform a theory-building approach. Specifically, this work examines several concepts related with entrepreneurial development and the influence of double- and triple-bottom-line agribusiness models, together with conditioning factors for obtaining responsible financing. Together with the model 
at issue, this work provides a duly supported conceptual approach with five associative propositions, which ultimately fosters the prospective testing of the model trough deductive methods in both ways: qualitative and quantitative.

Keywords: responsible entrepreneurship; socially responsible investments; entrepreneurial collaboration network; sustainability.

\section{INTRODUCCIÓN}

La inversión social responsable (ISR), se define como aquella asignación de recursos económicos sobre propuestas empresariales que, además de la intención legítima de generar riqueza, también tienen como propósito dar luz a iniciativas que busquen alcanzar los objetivos de sostenibilidad dentro de su actividad productiva (Betournay, 2015; Michelucci, 2017). Las ISRs, se concentran en buscar y en financiar proyectos que contribuyan a un cambio de paradigma, introduciendo en los negocios un enfoque más integral de bienestar colectivo o de triple impacto (Bugg-Levine \& Goldstein, 2009). Por consiguiente, sería lógico pensar que la ISR también contribuye a la construcción de desarrollo empresarial y al refuerzo de la competitividad empresarial; sin embargo, para que su adopción produzca los resultados esperados, el contexto empresarial respectivo, así como las políticas y los actores implicados, juegan un papel importante, a lo largo del proceso. Lamentablemente, la comprensión que hasta el momento se tiene sobre esta temática es aún muy primitiva $y$, en consecuencia, con inmensos vacíos conceptuales y teóricos (Clarkin \& Cangioni, 2016; Ormiston et al. 2015).

En este sentido, el propósito de una ISR en países desarrollados presenta un grado de orientación que ha evolucionado desde el apoyo tradicional desinteresado a fundaciones y proyectos sin ánimo de lucro, hasta la sofisticación financiera, propia de las inversiones en proyectos productivos rentables, con énfasis en la sostenibilidad (Nicholls, 2010). Sin embargo, en el contexto de economías en vía de desarrollo, se ha observado que una ISR podría también tener una incidencia significativa en aras de impulsar el desarrollo económico de las naciones y, por ende, el desarrollo de mejoras sociales asociadas a la creación oportunidades laborales y la consolidación de un tejido micro-empresarial, como impacto indirecto (SpiessKnafl \& Scheck, 2017).

El campo de los agronegocios, en particular y, más aún, en un contexto de una economía en desarrollo, no es la excepción. De hecho, es un rubro de especial atractivo para las ISR, por su misma naturaleza, relacionada con el desarrollo sostenible (Pons et al. 2012). El impacto social o medioambiental generado por este tipo de iniciativas es casi directo, llegando a abarcar grandes porcentajes de población con necesidades tangibles y crecientes para su bienestar y progreso. Complementariamente, las potencialidades existentes, en cuanto a diversificación de la oferta y la demanda, así como el despertar por conceptos, como el comercio justo, el mercado de lo orgánico y la sofisticación en los estilos de vida, hacen que muchos de los modelos de agronegocio concebidos, se puedan proyectar hacia su crecimiento y consolidación comercial y, por ende, financiera (Spiess-Knafl \& Scheck, 2017).

En esta línea, el presente trabajo plantea una aproximación basada en un modelo teórico idealizado, en donde se propone que la ISR actúe como agente dinamizador del desarrollo empresarial en los agronegocios de un país en vía de desarrollo. Para esto, además del análisis conceptual respectivo, la investigación se enmarca, esencialmente, en experiencias reales, que ayudan a entender las diferentes interacciones, desde la evidencia práctica en una nación específica (en este caso, Colombia). Así, se acude al planteamiento de proposiciones teóricas, a partir de un análisis de framework (Ritchie \& Spencer, 1994), conformado por casos prácticos reales de iniciativas emprendedoras, apoyadas por ISR. Para ello, se aclara que el contexto geográfico que sirve como insumo para dicho análisis es meramente indicativo poseedor de un carácter crítico, toda vez que, dada su dinámica y potencial, en lugar de describir o explorar, permitiría, eventualmente, confirmar, cambiar, modificar o ampliar el conocimiento, que ha sido producido a la fecha.

De este modo, a través de este trabajo de corte puramente teórico, se pretende ampliar el campo de conocimiento en torno a la ISR, sembrando la inquietud práctica de su utilización a diferentes niveles y contextos geográficos. En cuanto al modelo obtenido, se propone resaltar la viabilidad de promover e impulsar las iniciativas de sostenibilidad como parte integral de los agronegocios, mitigando, a través de esto, las necesidades insatisfechas relacionadas con la actividad productiva y, de esta forma, afrontar el reto global de generar valor agregado, con base en el doble y triple impacto.

El caso particular del sector agropecuario colombiano es de especial atención, ya que, junto con el de la inclusión financiera, es uno de los dos principales destinatarios presentes y futuros de ISR del país. Por un lado, Colombia es un país con diversidad de climas y con una ubicación privilegiada, capaz de concebir una oferta variada de productos agrí́colas, tanto para el mercado nacional como internacional. Por otro lado, este es un sector en donde, de una u otra forma, los inversores ya han desarrollado conocimiento práctico en la materia, lo que lo convierte en un rubro todavía más atractivo para la recepción de un mayor caudal de recursos (Dumont et al. 2016). Y es que se insinúa que Colombia tiene un potencial formidable de convertirse en un actor de clase mundial en este sector, con una idea centrada en el largo plazo, en donde los avances por lograr posibilitarían al país pasar de ser un simple proveedor de materias primas básicas a ser un productor de productos terminados de mayor valor agregado (Ramírez, 2013). Para ejemplificar, vale la pena mencionar la existencia de iniciativas, cuyo fin último está en la adopción de nuevos paradigmas energéticos complementados con la agricultura (Castiblanco et al. 2015) o propuestas de cooperación internacional en cultivos tradicionales, como el café (Gonzalez-Perez \& Gutierrez-Viana, 2012). Complementariamente, desde lo social, es innegable que el desarrollo en este sector tiene el potencial de crear empleos y generar mayores ingresos en las comunidades rurales, logrando una estabilidad necesaria para un crecimiento paulatino de las ISR (Dumont et al. 2016). 
La posibilidad de avanzar académicamente con respecto al presente tópico, se relaciona de manera simultánea con tres necesidades, aún pendientes por desarrollar: Primero, la identificación de la ISR como concepto integral que, junto con otras concepciones propias de otros dominios del conocimiento, permiten fortalecer un campo multifacético y bastante reciente, que pretende ilustrar una figura de naturaleza práctica, desde la perspectiva teórica (Höchstäder \& Scheck, 2015). Segundo, la carencia de aportes académicos enfocados en rubros específicos en aras de incrementar la calidad y la cantidad de trabajos relacionados con las ISR, teniendo en cuenta el escaso conocimiento que se tiene de ellas, así como su relevancia y pertinencia en el contexto de los países en vía de desarrollo (Contreras-Pacheco et al. 2017). Finalmente, se hace posible entender su aplicabilidad, a partir de la perspectiva receptora de una ISR (para este caso, asumir el lugar del emprendedor), lo cual, representa un tema que, modestamente, se ha tratado en la literatura científica a la fecha (Ormiston et al. 2015; Clarkin \& Cangioni, 2016). Evidentemente, la aproximación que de este aspecto se hará, contempla más la descripción, que aquellas particularidades normativas que evocan el "deber ser" y, por lo tanto, sugieren una propuesta idealizada del concepto.

\section{MATERIALES Y MÉTODOS}

El presente estudio hace uso de un análisis cualitativo, basado en un framework sobre siete casos empresariales presentes en el entorno particular de los agronegocios en Colombia. Dicha muestra de connotación teórica corresponde a igual número de proyectos empresariales de triple impacto que, naturalmente, fueron o están siendo beneficiarios de ISR y que, además, han logrado alcanzar cierto nivel de afianzamiento en sus respectivos negocios. Su selección responde al acervo disponible de los datos y se considera suficiente (i.e., saturación teórica), en la medida en que la interpretación del análisis acumulado de los casos, reporte resultados redundantes (Mason, 2010).

La herramienta denominada "análisis de framework" tiene sus fundamentos metodológicos en la propuesta de Ritchie \& Spencer (1994), con el objetivo de abordar el análisis cualitativo de teorías, tomando como puntos de partida el agrupamiento de casos de estudio. El análisis de framework representa una técnica de análisis conceptual flexible, en donde los datos recolectados son filtrados, esquematizados y clasificados en concordancia con las cuestiones principales, que son objeto de análisis (Srivastava \& Thomson, 2009). Sus objetivos van desde la mera descripción o interpretación de realidades hasta la generación de proposiciones teóricas, que ayudan a explicar los fenómenos asociados a dichas realidades (Ritchie \& Spencer, 1994). Así, el análisis en cuestión no corresponde a una validación empírica, ni intenta generar una comprensión definitiva sobre la temática a nivel general, sino que, en su lugar, se considera un vehículo para proponer una serie de situaciones posibles, ajustadas a un marco teórico específico. Adicionalmente, es de anotar que, a pesar de un entorno geográfico común, los casos seleccionados poseen un carácter y naturaleza diversa, lo cual, le brinda la dosis suficiente de homogeneidad y representatividad al estudio, facilitando la transferibilidad de las contribuciones obtenidas.

Las etapas de un análisis de framework (Ritchie \& Spencer, 1994) son básicamente cinco: i) familiarización con el contexto respectivo, ii) identificación de un marco conceptual y teórico apropiado para análisis, iii) indexación (o codificación) de los datos en bruto en función del marco conceptual seleccionado, iv) esquematización y compendio en aras de lograr una recomposición conceptual "como un todo" de los resultados y, finalmente, v) interpretación de los datos, que es donde, dependiendo del caso, se pueden definir conceptos, crear tipologías, desarrollar estrategias e, incluso, como aquí se propone, encontrar asociaciones entre constructos.

Para iniciar el proceso, se procede a entender el contexto (que, como ya se mencionó, es de naturaleza indicativa) y a los actores que intervienen de una forma activa en el otorgamiento de ISR, a las empresas en cuestión. Asimismo, junto con el establecimiento del marco conceptual relacionado con la ISR, se acude a fuentes de referencias históricas y descriptivas, así como a consultas en dominios y en comunicaciones corporativas de las empresas escogidas y de medios de comunicación convencionales. También, se procedió con el análisis de algunas entrevistas informales a algunos actores implicados en el proceso de inversión y de apalancamiento sobre las compañías tratadas y del estudio de algunos reportes y relatos de los emprendedores, sobre sus experiencias puntuales.

En primera instancia, se contemplaron 11 casos diferentes; sin embargo, dependiendo de la disponibilidad de información, fueron finalmente depurados a siete. Por ello, hechos como la concentración de los mismos en un fondo de inversión específico o la exclusión de regiones particulares de la geografía colombiana son elementos que escapan de los criterios de selección para la muestra particular (Cuadro 1).

En cuanto a las etapas finales del análisis efectuado, se compilan una serie de ideas extraídas del análisis independiente de cada caso particular, en aras de hacer la data comparable. De esta manera, se ponen en perspectiva sus propósitos de negocio, composición de redes de apoyo, condicionantes para el acceso a ISR e impactos logrados a la fecha. Para ello, es fundamental asumir que una dinámica de penetración de este tipo de inversiones en economías en vía de desarrollo, crea un importante chance de proliferación en su aceptación (Stagars, 2015).

En el cuadro 2, se presenta una síntesis del framework en cuestión, en donde se ve representado el proceso de compendio. 
Cuadro 1. Framework - Generalidades.

\begin{tabular}{|c|c|c|c|c|c|c|c|c|}
\hline$\#$ & Nombre & Región & Año & $\begin{array}{l}\text { Fuentes de Consulta } \\
\text { (Adicional a páginas web, } \\
\text { blogs y redes sociales) }\end{array}$ & $\begin{array}{l}\text { Tipo de } \\
\text { Negocio }\end{array}$ & Modelo de Negocio & $\begin{array}{c}\text { Fondo de } \\
\text { Impacto }\end{array}$ & Sponsor \\
\hline 1 & Fresota & Cauca & 2000 & Camacho (2014) & $\begin{array}{l}\text { Cooperativa de } \\
\text { Agricultores }\end{array}$ & $\begin{array}{l}\text { Cultivo y comerciali- } \\
\text { zación de fresas }\end{array}$ & $\begin{array}{l}\text { IC Fun- } \\
\text { dación }\end{array}$ & Smurfit Kappa \\
\hline 2 & Huertaap & $\begin{array}{l}\text { Bogotá, } \\
\text { D.C. }\end{array}$ & 2017 & Pulsosocial.com (2017) & $\begin{array}{l}\text { Sociedad Mer- } \\
\text { cantil }\end{array}$ & $\begin{array}{l}\text { Aplicación móvil para } \\
\text { despacho de frutos } \\
\text { orgánicos }\end{array}$ & $\begin{array}{l}\text { Athena } \\
\text { Impacto }\end{array}$ & $\mathrm{N} / \mathrm{A}$ \\
\hline 3 & $\begin{array}{l}\text { Guayabas } \\
\text { Veleñas }\end{array}$ & Santander & 2011 & Muñoz et al. (2017) & $\begin{array}{l}\text { Cooperativa de } \\
\text { Agricultores }\end{array}$ & $\begin{array}{l}\text { Cultivo y manufactura } \\
\text { de guayabas y produc- } \\
\text { tos derivados }\end{array}$ & $\begin{array}{l}\text { IC Fun- } \\
\text { dación }\end{array}$ & $\begin{array}{l}\text { Corporación } \\
\text { Vélez } 500 \text { años }\end{array}$ \\
\hline 4 & Fruandes & Tolima & 2002 & Fruandes (2018) & $\begin{array}{l}\text { Sociedad Mer- } \\
\text { cantil }\end{array}$ & $\begin{array}{l}\text { Comercialización de } \\
\text { frutas orgánicas (fres- } \\
\text { cas y secas) }\end{array}$ & $\begin{array}{l}\text { Fondo } \\
\text { Inversor }\end{array}$ & $\mathrm{N} / \mathrm{A}$ \\
\hline 5 & Corseda & Cauca & 1999 & Garzón (2015) & $\begin{array}{l}\text { Sociedad Mer- } \\
\text { cantil }\end{array}$ & $\begin{array}{l}\text { Producción y comer- } \\
\text { cialización de seda } \\
\text { y productos de seda } \\
\text { orgánica certificada } \\
\text { (únicos en el mundo) }\end{array}$ & $\begin{array}{l}\text { IC Fun- } \\
\text { dación }\end{array}$ & $\mathrm{N} / \mathrm{A}$ \\
\hline 6 & $\begin{array}{l}\text { Chocolate } \\
\text { Colombia }\end{array}$ & $\begin{array}{l}\text { Antioquia } \\
\text { - Córdoba }\end{array}$ & 2014 & Ospina (2016) & $\begin{array}{l}\text { Agrupación de } \\
\text { asociaciones } \\
\text { productoras }\end{array}$ & $\begin{array}{l}\text { Cultivo, producción } \\
\text { y comercialización } \\
\text { de cacao y productos } \\
\text { derivados }\end{array}$ & $\begin{array}{l}\text { IC Fun- } \\
\text { dación }\end{array}$ & $\mathrm{N} / \mathrm{A}$ \\
\hline 7 & Asapibas & $\begin{array}{c}\text { Antioquia } \\
\text { - Bolívar }\end{array}$ & 2013 & Contexto ganadero (2018) & $\begin{array}{l}\text { Empresa Aso- } \\
\text { ciativa }\end{array}$ & Miel de Abejas & $\begin{array}{l}\text { IC Fun- } \\
\text { dación }\end{array}$ & $\begin{array}{l}\text { Grupo Mineros } \\
\text { S.A. - USAID }\end{array}$ \\
\hline
\end{tabular}

Cuadro 2. Análisis de Framework ISR - Compendio.

\begin{tabular}{|c|c|c|c|c|}
\hline \# & Propósito Social Explícito & $\begin{array}{l}\text { Red de Apoyo } \\
\text { (Involucrados) }\end{array}$ & $\begin{array}{c}\text { Condicionantes para } \\
\text { Acceso a ISR }\end{array}$ & $\begin{array}{l}\text { Impacto Social de } \\
\text { Largo Plazo }\end{array}$ \\
\hline 1 & $\begin{array}{l}\text { Mejorar la calidad de vida y el bienestar del asociado } \\
\text { y de su núcleo familiar, a través de la generación } \\
\text { de valor agregado en la cadena productiva de la } \\
\text { fresa y de la prestación de servicios comunitarios, } \\
\text { contribuyendo al desarrollo económico y social de } \\
\text { la comunidad }\end{array}$ & $\begin{array}{l}\text { Sponsor. } 100 \text { familias campesinas } \\
\text { asociadas. Inversionista }\end{array}$ & $\begin{array}{l}\text { Compromiso del sponsor, } \\
\text { experiencia, asociatividad }\end{array}$ & $\begin{array}{l}\text { Supervivencia. Bienestar } \\
\text { de región }\end{array}$ \\
\hline 2 & $\begin{array}{l}\text { Mejorar la calidad de vida de campesinos, a través } \\
\text { de los principios de comercio justo y asegurar } \\
\text { tierras productivas a largo plazo, promoviendo la } \\
\text { agricultura orgánica }\end{array}$ & $\begin{array}{l}120 \text { familias campesinas proveedo- } \\
\text { ras. Inversionista }\end{array}$ & $\begin{array}{l}\text { Tecnología, espíritu de } \\
\text { equipo }\end{array}$ & $\begin{array}{l}\text { Promoción de agricultu- } \\
\text { ra orgánica. Principios de } \\
\text { comercio justo. Bienestar } \\
\text { de región }\end{array}$ \\
\hline 3 & $\mathrm{~N} / \mathrm{A}$ & $\begin{array}{l}\text { Sponsor. } 5 \text { asociaciones de pro- } \\
\text { ductores - } 51 \text { productores de base. } \\
\text { Universidad y grupos de apoyo. } \\
\text { Inversionista }\end{array}$ & $\begin{array}{l}\text { Asociatividad, apoyo ofi- } \\
\text { cial y privado, experiencia, } \\
\text { perseverancia }\end{array}$ & $\begin{array}{l}\text { Supervivencia. Bienestar } \\
\text { de región }\end{array}$ \\
\hline 4 & $\begin{array}{l}\text { Generar un ecosistema de bienestar social, huma- } \\
\text { no y medio-ambiental derivado de la rentabilidad } \\
\text { de los negocios emprendidos }\end{array}$ & $\begin{array}{l}350 \text { familias productoras. Gremi- } \\
\text { os. ONGs. Inversionistas }\end{array}$ & $\begin{array}{l}\text { Marketing, enfoque inter- } \\
\text { nacional, portafolio dif- } \\
\text { erenciado de productos, } \\
\text { experiencia, liderazgo }\end{array}$ & $\begin{array}{l}\text { Proveeduría de productos } \\
\text { saludables. Promoción de } \\
\text { agricultura orgánica. Prin- } \\
\text { cipios de comercio justo. } \\
\text { Bienestar de región }\end{array}$ \\
\hline
\end{tabular}


Continuación cuadro 2.

\begin{tabular}{|c|c|c|c|c|}
\hline 5 & $\begin{array}{l}\text { Mejorar el nivel de vida de las familias caucanas } \\
\text { dedicadas al arte de la seda, mediante el fortale- } \\
\text { cimiento de la actividad, desde la cría del gusano } \\
\text { hasta la comercialización de tejidos en los mercados } \\
\text { nacionales e internacionales }\end{array}$ & $\begin{array}{l}200 \text { familias productoras. Gobier- } \\
\text { no Nacional y departamental. In- } \\
\text { versionista }\end{array}$ & $\begin{array}{l}\text { Producto único, cono- } \\
\text { cimiento, asociatividad, } \\
\text { apoyo oficial, experiencia } \\
\text { y perseverancia }\end{array}$ & $\begin{array}{l}\text { Promoción de agricultura } \\
\text { y consumo de productos } \\
\text { orgánicos. Principios de } \\
\text { comercio justo. Bienestar } \\
\text { de región }\end{array}$ \\
\hline 6 & $\begin{array}{l}\text { Aunar esfuerzos que propendan por el fortaleci- } \\
\text { miento de las organizaciones productoras de cacao } \\
\text { del área de influencia, mediante la implementación } \\
\text { de estrategias y de técnicas comerciales, que bus- } \\
\text { quen la consolidación del agronegocio del cacao, } \\
\text { sus subproductos y demás actividades del sector } \\
\text { agropecuario }\end{array}$ & $\begin{array}{l}\text { Gobierno Nacional. } 8 \text { asociaciones } \\
\text { productoras. Familias campesinas. } \\
\text { Inversionista }\end{array}$ & $\begin{array}{l}\text { Asociatividad, diversifi- } \\
\text { cación de productos, ex- } \\
\text { periencia, certificaciones } \\
\text { internacionales, apoyo pú- } \\
\text { blico y privado }\end{array}$ & $\begin{array}{l}\text { Promoción de consumo } \\
\text { de productos nacionales } \\
\text { en el exterior. Principios } \\
\text { de comercio justo. Bien- } \\
\text { estar de región }\end{array}$ \\
\hline 7 & $\begin{array}{l}\text { Unir y representar a los productores y grupos inte- } \\
\text { resados del sector apícola de la región de influencia }\end{array}$ & $\begin{array}{l}\text { Sponsor. } 33 \text { apicultores. Gremios. } \\
\text { Inversionista }\end{array}$ & $\begin{array}{l}\text { Asociatividad, experiencia, } \\
\text { apoyo oficial y privado }\end{array}$ & $\begin{array}{l}\text { Supervivencia. Bienestar } \\
\text { de región }\end{array}$ \\
\hline
\end{tabular}

\section{RESULTADOS Y DISCUSIÓN}

Independientemente del rubro económico específico, una operación de ISR, se lleva a cabo con el propósito de emprender proyectos empresariales de naturaleza responsable; esto quiere decir que, en cualquier etapa del ciclo de vida de una compañía determinada, la ISR tiene la capacidad de promover globalmente las iniciativas sociales o medio-ambientales de una nación o de un territorio. La propuesta subsecuente consiste en un análisis minucioso, a través del lente del framework presentado.

De esta forma, se parte de (i) y (ii):

(i) ¿Cuáles son los condicionantes de la ISR en el contexto de los agronegocios en Colombia? Cada una de las empresas analizadas tiene un origen o un crecimiento, que ha sido soportado por ISR en diferente medida. Se pueden apreciar elementos, como el liderazgo, el capital humano, las instituciones financieras, el respaldo institucional de un sponsor o la existencia de un mercado natural, propicio para el crecimiento, claves para construir la confianza del inversionista, para alcanzar la decisión de apostar por estos proyectos; es decir que, en la medida en que se dieron las condiciones para satisfacer las circunstancias filantrópicas o financieras, se están dando las condiciones para estimular el fenómeno de la ISR en ese sector. Adicionalmente y no menos importante, a medida que la ISR hace parte de la creación y crecimiento de las diversas iniciativas empresariales, con doble o triple propósito, se van aprovechando las oportunidades para crear un entorno emprendedor más colaborativo.

Es evidente que, para los casos analizados, el espíritu asociativo de los emprendedores y, al mismo tiempo, la obtención de beneficios otorgados por actores externos fue fundamental para la puesta en marcha y posterior desarrollo de los agronegocios analizados. De hecho, en todos los casos, dicho espíritu se vio acrecentado por la obtención de los beneficios asociados a la ISR respectiva. Cooperativas (casos 1 y 3), redes asociativas de productores (casos
7 y 8) o sociedades mercantiles convencionales (casos 2, 4 y 5), son ejemplos de lo anterior. Así, de una forma u otra, dichas "redes de apoyo" fueron necesarias para propiciar la búsqueda y la posterior consecución de la ISR requerida y, a su vez, esta consecución terminó estimulando el fortalecimiento de dichas redes. De esta forma, la lógica nos sugiere que, si estas redes fueron construidas gracias al esfuerzo propio de los equipos emprendedores respectivos sin una formalidad específica, se puede esperar que, en un escenario de un real establecimiento de relaciones institucionales, los resultados sean más que sobresalientes en cantidad, en calidad de los negocios apoyados y en monto de inversiones. En conclusión, de manera simultánea se sugiere que:

Proposición 1: La existencia previa de adecuadas redes colaborativas de emprendimiento orientadas a modelos de agronegocio de triple impacto influye en el fomento de la ISR en una economía y,

Proposición 1': La dinámica generada por la ISR estimula el desarrollo y la consolidación de redes colaborativas de emprendimiento orientadas a modelos de agronegocio de triple impacto.

(ii) ¿Cuál es el resultado natural a largo plazo de dicho impulso sobre una sociedad? Así como fue afirmado por Cohen (2014), la conducta derivada de este fenómeno es similar al de las demás inversiones, aunque con la diferencia de estar orientada a compañías, cuya meta sigue siendo de corte mercantilista, con una misión empresarial responsable. De esta forma, su dinamización generaría un resultado final compatible con el significado de progreso social. Esto, se da por la vía de la concienciación de los emprendedores hacia el compromiso, no solo de la retribución sobre el capital obtenido, sino también, a través de la implementación de una visión a largo plazo, que trasciende la rentabilidad financiera. Cada uno de los casos analizados es coincidente con dicha aproximación; de hecho, sus propósitos se relacionan directamente con el impacto 
positivo hacia sus stakeholders $y$, por encadenamiento de ideas, con el impacto positivo hacia su entorno productivo.

En una de las manifestaciones más relevantes del presente trabajo, se evidencia que la materialización de una operación de ISR sobre un negocio es mucho más lo que se obtiene, que el mero capital que interviene en dicha transacción. Algunos de los verdaderos valores agregados de este fenómeno vienen también en forma de crecimiento sostenible de otros negocios impactados, por parte del negocio receptor de la inversión. En los casos de cooperativas y redes productivas, por ejemplo, es notable cómo sus modelos de negocio brindan la posibilidad de participación y de creación de riqueza a aquellos pequeños emprendedores, familias campesinas o negocios de las diferentes regiones rurales colombianas, mediante un esquema asociativo. Asimismo, la participación de proveedores, empleados y comunidades circundantes posibilita la irrigación de beneficios a otros actores de las cadenas productivas analizadas. Por estas razones, la idea planteada sería de tal forma que:

Proposición 2: El fenómeno de la ISR ayuda a dinamizar la formación de un desarrollo empresarial orientado a modelos de agronegocio de triple impacto.

De manera adicional, al poner en perspectiva los constructos respectivos con sus relaciones iniciales, se hace notorio que hay un par de efectos que enriquecen el modelo teórico propuesto, haciéndolo más explicativo de la realidad. Primero, se sugiere que el efecto generado por la existencia y la operación adecuada de una red colaborativa de emprendimiento sostenible en agronegocios no solo crea un mayor dinamismo de la figura de la ISR, sino que también tenga un efecto todavía más trascendental en términos de sostenibilidad sobre el desarrollo del aparato productivo de una sociedad, incluso, sin la existencia de esta última.

La lógica es que dicha dinámica envía señales, en donde se muestra un ambiente colectivo propicio para el impulso de agronegocios, generadores de doble y triple impacto. Una posible explicación a este fenómeno es que se genera una especie de "aglomeración accidental sostenible" de compañías (tipo clúster) que, al atraer inversión directa, puede implicar, según Gugler \& Brunner (2007), que se logre un efecto lineal, que llega a mejorar la competitividad de una nación, por vías de la competitividad de sus empresas.

Así pues, la propuesta complementaria es que:

Proposición 3: La existencia previa de adecuadas redes colaborativas orientadas al emprendimiento de modelos de agronegocio de triple impacto influye en la consolidación y el fortalecimiento del desarrollo empresarial sostenible de la sociedad.

Por otra parte, pese a que es claro que un mejor ritmo en la adopción de la figura de la ISR, representa una mejora sustancial del sistema productivo en su conjunto, también se puede asegurar que lo segundo influye en lo primero. Lo anterior, se debe, principalmente, a que existe una fuerte evidencia en torno a que un ambiente positivo para hacer y desarrollar negocios redunda inevitablemente en una mayor inversión para los mismos y para nuevos agronegocios. En otras palabras, un entorno sostenible competitivo tendrá que atraer "inversionistas responsables", por lo que, por definición, se sugeriría también que:

Proposición 2': Un desarrollo empresarial sostenible orientado a modelos de agronegocio de triple impacto trae consigo un mayor flujo de operaciones de ISR a la economía de un país.

De esta forma, la figura 1 detalla integralmente la aproximación teórica asociada con el anterior análisis.

Recapitulando, el presente trabajo se preguntó por aquellos antecedentes que impulsan la dinámica de la ISR (Combs, 2014; Betournay, 2015; Höchstäder \& Scheck, 2015), así como sus resultados en el largo plazo, haciendo uso del contexto de los agronegocios en Colombia. Para ello, se partió de una aproximación conceptual, conducente a un modelo teórico ideal, que siguió una metodología de desarrollo de teorías, tomando como referencia un análisis de framework (Ritchie \& Spencer, 1994), sobre siete casos de estudio. De esta forma, se lograron identificar los elementos suficientes para proponer tres variables latentes: i) las Redes Colaborativas de Triple Impacto, ii) la ISR y, iii) el Desarrollo Empresarial Sostenible y su relación a través de cinco proposiciones de asociación, las cuales, pretenden ayudar a explicar los fenómenos reales a partir de la teoría. Algunas circunstancias propias del contexto estudiado hicieron parte de la aproximación conceptual propuesta.

Como resultado de esta investigación, se logra ilustrar, de manera sistémica, la forma en que una ISR -como mecanismo de financiamiento de agronegocios- puede llegar a ser un agente dinamizador de alto impacto en la sociedad y, por lo tanto, una alternativa atractiva, cuando las políticas público-privadas buscan mejorar el desarrollo empresarial en países en vía de desarrollo. La más relevante contribución del presente resultado corresponde a la propuesta del modelo teórico respectivo, que construye conocimiento sobre lo producido, a la fecha, dentro de los tópicos aquí tratados. Dicha aproximación sugiere que las ISR sobre agronegocios impulsan y son impulsadas también por un ecosistema empresarial (red colaborativa), orientado a estimular modelos de agronegocio de doble y triple impacto. Estos elementos tienen la capacidad de promover y ser promovidos por la existencia y operación de un desarrollo empresarial sostenible, que se encargue de fortalecer la experiencia en nuevas iniciativas y proyectos en el sector de los agronegocios. Aspectos, como la capacidad del equipo emprendedor y el propósito específico del negocio en cuestión, son vitales para propiciar esta asociación.

De la misma manera, se sugiere que los países en vía de desarrollo, como el caso de Colombia, poseen las condiciones necesarias para generar y atraer un mayor nivel de ISR sobre iniciativas en agronegocios, con el objetivo de forjar un más estable desarrollo para sus pueblos. Muy a pesar de su contundencia en un entorno práctico, dicho concepto ha estado surgiendo de manera paulatina en la literatura organizacional, sugiriendo que los nuevos emprendimientos responsables cuentan con una alternativa de 


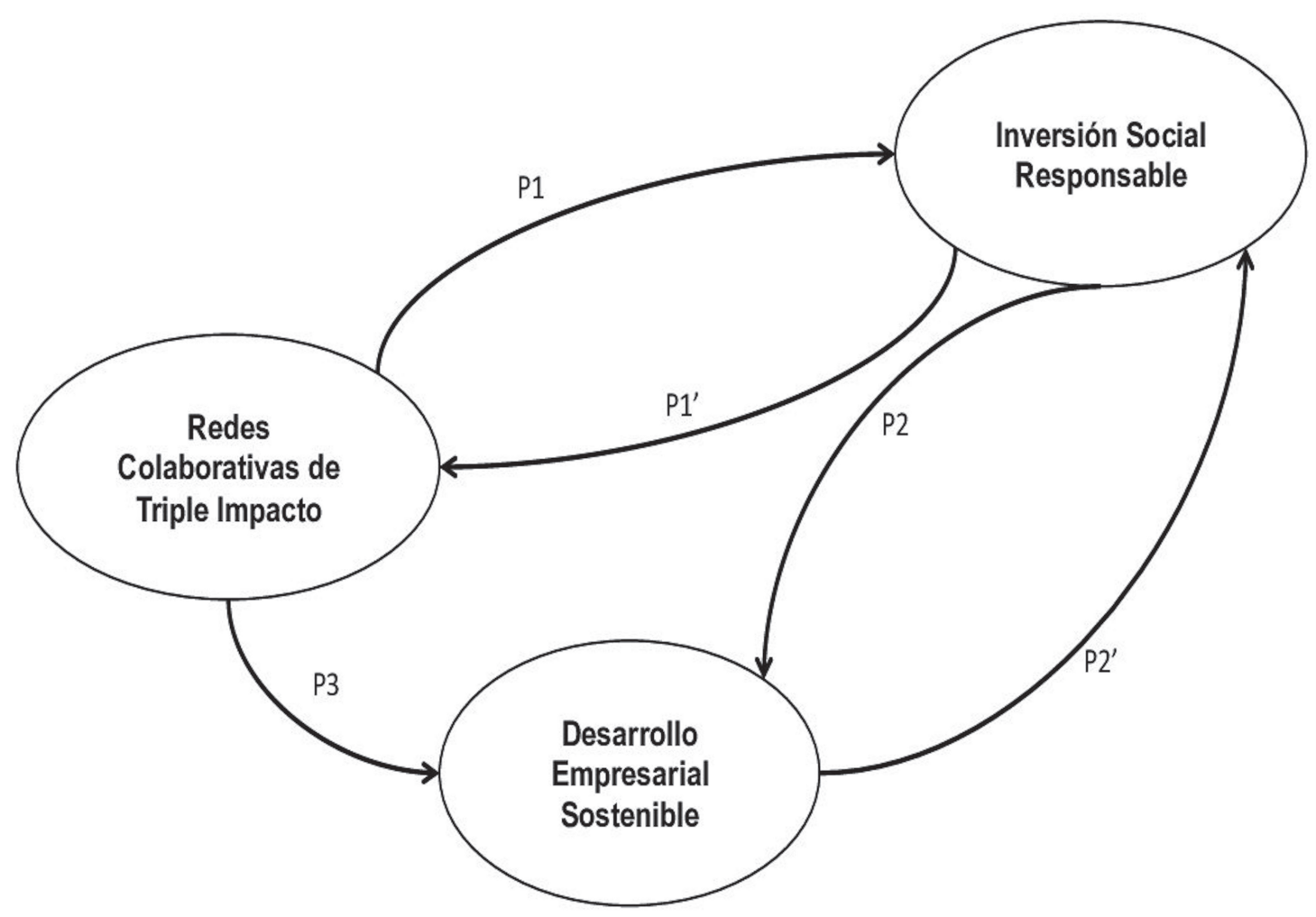

Figura 1. Modelo teórico propuesto.

financiamiento, que trasciende una simple evaluación financiera de la rentabilidad y del riesgo, como lo sugiere la teoría clásica, sino que también abogan por un propósito sostenible con su accionar. En otras palabras, que dichas inversiones están enfocadas a asumir posiciones de riesgo crediticio en aras de apoyar el desarrollo de emprendimientos sostenibles. Lo anterior, se sustenta también en que, adicional al espíritu filantrópico de los inversionistas respectivos, interesantemente, se han encontrado resultados financieros positivos, gracias al rendimiento de estas operaciones (Bugg-Levine \& Goldstein, 2009; Clarkin \& Cangioni, 2016).

Un elemento que fue tímidamente considerado, pero que es evidente dentro del análisis presentado, tiene que ver con las características del equipo emprendedor, así como el enfoque estratégico o innovador de los agronegocios abordados. Sin demeritar algunos casos examinados, se podría inferir que, dichos elementos, son decisivos por cuenta de los resultados empresariales alcanzados y proyectados, especialmente, en las sociedades mercantiles. Lamentablemente, el nivel de desarrollo autónomo de algunas cooperativas y empresas asociativas evaluadas de manera aislada, es aún incipiente, lo que lleva a concentrar, en exclusiva, el concepto de la ISR sobre los impactos sociales o medio-ambientales generados. Lo anterior, a pesar de parecer medianamente válido (Bugg-Levine \& Goldstein, 2009), riñe con el concepto de triple impacto (Elkington, 1994) y puede atentar con la sostenibilidad en el largo plazo de los negocios (Nicholls, 2010). Bajo esas condiciones, el modelo propuesto correría peligro y los verdaderos objetivos de la ISR no se podrían alcanzar. Se sugiere que, con el ánimo de complementar lo expuesto anteriormente, un análisis más profundo de estos fenómenos es conveniente y necesario para refinar ideas y establecer diferentes niveles de conocimiento sobre la temática en cuestión.

Sin lugar a dudas, el ambiente de los agronegocios en un país en desarrollo, como Colombia, implica el aprovechamiento de grandes oportunidades, pero, a la vez, significa asumir grandes retos y enfrentar obstáculos. Por una parte, las ventajas derivadas de su ubicación, climas, capacidad laboral de su gente y variedad geográfica, tienen el potencial de abrir la puerta al apetito inversionista de aquellos que buscan una adecuada amalgama entre rentabilidad y riesgo. Por otra parte, la enorme desigualdad social existente hace que se evidencie una importante cantidad de necesidades insatisfechas en la población rural, quienes, en últimas, son un grupo de interés clave para garantizar el éxito de los proyectos emprendidos. El presente trabajo defiende la idea que, en cierta forma, la ISR -adecuadamente ejecutada- hace posible que esta dualidad sea superada y que el esquema finalmente logrado sea de generación conjunta de valor. Los casos presentados, en mayor o menor medida, son ejemplos de lo anterior y aunque algunos de ellos estén en mora de demostrar un mejor desempeño empresarial, la tenacidad y la motivación de los promotores de las iniciativas presentadas anteriormente, merece un reconocimiento especial, digno de análisis y de replicación. 
A pesar de hacer claridad previa con respecto al carácter conceptual y no empírico del trabajo, hay algunas limitaciones que se deben subrayar. En primera instancia, se resalta la circunscripción del análisis a un contexto geográfico y económico particular. Adicionalmente, también se deja constancia de la incapacidad de generalización de las conclusiones obtenidas. Al no ser un ejercicio de exploración sobre una muestra representativa, lo que sigue sería intentar probar dichas proposiciones de manera deductiva. A futuro, se espera, precisamente, que estas líneas sirvan como marco de trabajo para estudios posteriores, con el propósito de convertir las proposiciones en hipótesis y trabajar sistemáticamente sobre ellas. De cualquier forma, el debate académico queda planteado, ya que el conocimiento sobre este tipo de temáticas está por desarrollar, de tal manera, que cualquier aporte agregado debería ser bienvenido para contribuir al entendimiento de la realidad.

Finalmente, las implicaciones prácticas que se derivan del presente trabajo podrían incluir sendas recomendaciones para los emprendedores deseosos por hacer crecer sus negocios respectivos (especialmente, en el sector agro-industrial); para los administradores de fondos de inversión o inversionistas, quienes buscan nuevas alternativas de diversificación en sus portafolios o que simplemente les llama la atención el hecho de invertir responsablemente y, por supuesto, para todos los interesados en que el desarrollo empresarial de una sociedad crezca y se consolide sostenidamente, como representantes de gobiernos, gremios económicos, entidades educativas, cámaras de comercio y empresas de consultoría.

A manera de conclusión, el presente trabajo reivindica, desde un punto de vista netamente teórico, la necesidad de diseñar y de aplicar estructuras más efectivas de colaboración, que permitan el adecuado fomento de más y mejores proyectos emprendedores en el sector de los agronegocios, en cualquier contexto geográfico. Se propone, por tanto, que dicho impulso podría generar un impacto directo hacia un escenario real de desarrollo empresarial sostenible.

Conflicto de intereses: El manuscrito fue preparado y revisado con la participación de ambos autores, quienes declaramos que no existe ningún conflicto de intereses que ponga en riesgo la validez de los resultados presentados. Financiación: Estudio financiado por el grupo de investigación Finance \& Management de la Escuela de Estudios Industriales y Empresariales de la Universidad Industrial de Santander.

\section{REFERENCIAS}

1. BETOURNAY, M. 2015. Impact Investing. Call it what you will, some investors seek more than just financial returns. MidstreamBusiness. p.79-81.

2. BUGG-LEVINE, A.; GOLDSTEIN, J. 2009. Impact investing: harnessing capital markets to solve problems at scale. Community Development Investment Review. 2:30-41.

3. CAMACHO, J. 2014. Una Experiencia Muy Jugosa. In: Camacho, J.; Prata, L.; Jorge, S. (eds.), Empresas y Comunidades que
Transforman América Latina. Bogotá D.C.: Corporación RedEAmérica. p.42-57.

4. CASTIBLANCO, C.; MORENO, A.; ETTTER, A. 2015. Impact of policies and subsidies in agribusiness: The case of oil palm and biofuels in Colombia. Energy Economics. 49:676678. https://doi.org/10.1016/j.eneco.2015.02.025

5. CLARKIN, J.E.; CANGIONI, C.L. 2016. Impact Investing: A Primer and Review of the Literature. Entrepreneurship Res. J. 6(2):135-173. https://doi.org/10.1515/erj-2014-0011

6. COHEN, R. 2014. Impact Investment: The Invisible Heart of Markets (Rep.). Disponible desde Internet en https:// www.csi.uni-heidelberg.de/downloads / Impact $\% 20$ Investment $\% 20$ Report $\% 20$ FINAL.pdf [con acceso el $8 / 11 / 2015]$.

7. COMBS, K. 2014. More than Just a Trend: The Importance of Impact Investing. Corporate Finance Review. 18(6):12.

8. CONTEXTO GANADERO 2018. Se fortalece la apicultura en el Bajo Cauca. Disponible desde Internet en http:// contextoganadero.com/agricultura/se-fortalece-laapicultura-en-el-bajo-cauca [con acceso el 19/06/2018].

9. CONTRERAS-PACHECO, O.E.; PEDRAZA, A.C.; MARTÍNEZ, M.J. 2017. La inversión de impacto como medio de impulso al desarrollo sostenible: una aproximación multicaso a nivel de empresa en Colombia. Estudios Gerenciales. 33(142):13-23. https://doi.org/10.1016/j. estger.2017.02.002

10. DUMONT, K.; EDENS, G.; DE MARIZ, F.; ROCHA, R.; ROMAN, E.; ROSSI, A.; VALENCIA, N. 2016. The Impact Investing Landscape in Latin America. ANDE, LAVCA e IGT Impact Ventures. Disponible desde Internet en https://lavca.org/impact-investing-rep/el-panoramade-la-inversion-de-impacto-en-america-latina/ [con acceso el 20/02/2017].

11. ELKINGTON, J. 1994. Towards the sustainable corporation: Win-win-win business strategies for sustainable development. California Management Review. 36(2):90100. http://dx.doi.org/10.2307/41165746

12. FRUANDES. 2018. Impact Report 2017(Rep.). Disponible desde Internet en: https://fruandes.com/assets/ FRUANDES-INFORME-IMPACTO.pdf [con acceso el 19/06/2018].

13. GARZÓN, L.M. 2015. Identificación de líneas de investigación biotecnológicas para la sericultura en Colombia. Monografía de Especialización. UNAD. Popayán. p. 56. 
14. GONZALEZ-PEREZ, M.A.; GUTIERREZ-VIANA, S. 2012. Cooperation in coffee markets: the case of Vietnam and Colombia. Journal of Agribusiness in Developing and Emerging Economies. 2(1):57-73. https://doi. org/10.1108/20440831211219237

15. GUGLER, P.; BRUNNER, S. 2007. FDI effects on national competitiveness: A cluster approach. Internal Advances in Economic Research. 13(3):268-284. https://doi. org/10.1007/s11294-007-9091-1

16. HÖCHSTÄDER, A.K.; SCHECK, B. 2015. What's in a name: An analysis of impact investing understandings by academics and practitioners. Journal of Business Ethics. 132(2):449-475. http://doi.org/10.1007/s10551-014-23270

17. MASON, M. 2010. Sample Size and Saturation in PhD Studies Using Qualitative Interviews. Forum Qualitative Sozialforschung 11(3):8. http:/ / dx.doi.org/10.17169/fqs11.3.1428

18. MICHELUCCI, F.V. 2017. Social Impact Investments: Does an alternative to the anglo-saxon paradigm exist? VOLUNTAS: International Journal of Voluntary and Nonprofit Organizations. 28(6):2683-2706. https://doi. org/10.1007/s11266-016-9783-3

19. MUÑOZ, A.J.; PEÑA, Y.Y.; VARGAS, A. 2017. El rol de la asociatividad en la denominación de origen del bocadillo veleño colombiano como estrategia de competitividad. I+D Revista de Investigaciones. 9(2):27-37. https://doi. org/10.33304/revinv.v09n1-2017003

20. NICHOLLS, A. 2010. The institutionalization of social investment: The interplay of investment logics and investor. J. Social Entrepreneurship. 1(1):70-100. https:// doi.org/10.1080/19420671003701257

21. ORMISTON, J.; CHARLTON, K.; DONALD, M.S.; SEYMOUR, R.G. 2015. Overcoming the challenges of impact investing: insights from leading investors. J. Social Entrepreneurship. 6(3):352-378. https://doi.org/10.1080 /19420676.2015.1049285

22. OSPINA, G. 2016. El cacao, la opción en el Paramillo para dejar la coca. Disponible desde Internet en: http://www. elcolombiano.com/antioquia/el-cacao-la-opcion-en-elparamillo-para-dejar-la-coca-HA5570090 [con acceso el 19/06/2018].

23. PONS, E.; LONG, M.-A.; POMARES, R. 2012. Promoting Sustainable Food Systems Through Impact Investing. Washington, DC. 58p.

24. PULSOSOCIAL.com. 2017. Huerta app, la app que lleva del campo a tu mesa con un click. Disponible desde Internet en: https://pulsosocial.com/2017/07/11/huertaap-delcampo-a-tu-mesa-con-un-clic/ [con acceso el 19/06/2018]

25. RAMÍREZ, E. 2013. La competitividad de los agronegocios en Colombia: una reflexión académica. Magazín Empresarial. 9(22):29-34.

26. RITCHIE, J.; SPENCER, L. 1994. Qualitative data analysis for applied policy research. In: Bryman, A.; Burgess, R.G. (eds.), Analyzing Qualitative Data. London: Routeledge. p.173-194.

27. SPIESS-KNAFL, W.; SCHECK, B. 2017. Impact Investing: Instruments, Mechanisms and Actors. 171p. Palgrave Macmillan. https://doi.org/10.1007/978-3-319-66556-6

28. SRIVASTAVA, A.; THOMSON, S.B. 2009. Framework analysis: A qualitative methodology for applied policy research. Journal of Administration \& Governance. 4(2):72-79.

29. STAGARS, M. 2015. Impact Investment: Where Are We Now? In Impact Investment Funds for Frontier Markets in Southeast Asia: Creating a Platform for Instit. Capital, High-Quality Foreign Direct Investment, and Proactive Policy Making. London: Palgrave. p.6-45 